\title{
Calidad fisiológica y energía de germinación de semillas de balsamina (Momordica charantia L.)
}

Physiological quality and germination energy of bitter gourd (Momordica charantia L.) seeds

Fernando Barraza A $^{1}$; Orlando Benavides $\mathrm{B}^{2}$.; Francisco Torres $\mathbf{M}^{3}$.

1. Docente, I.A., Ph.D. Universidad de Córdoba, Montería, Colombia, barraza@fca.edu.co.

2. Docente, I.A. M.Sc. Universidad de Nariño, Pasto, Colombia, orlando.benavides2@gmail.com.

3. Docente, I.A. Ph.D. Universidad de Nariño, Pasto, Colombia, franjatm@hotmail.com.

Citar: BARRAZA, F.; BENAVIDES, 0.; TORRES, F. 2016. Calidad fisiológica y energía de germinación de semillas de balsamina (Momordica charantia L.). Rev.Cienc. Agri. 33(1):43 - 52.

Fecha de recepción: Agosto 20 de $2015 . \quad$ Fecha de aceptación: Febrero 16 de 2016.

\begin{abstract}
RESUMEN
Las semillas de balsamina presentan dificultad en la germinación debido a la testa gruesa y dura que rodea al embrión. El objetivo fue evaluar el efecto que tiene la imbibición de semillas de balsamina en agua sobre la energía de germinación, velocidad absoluta de germinación e índice de germinación. Se utilizó diseño experimental completamente al azar con tres tratamientos: T0 (control, sin imbibición en agua), T1 (semillas embebidas en agua a $22^{\circ} \mathrm{C}$ durante 24 horas) y T2 (semillas embebidas en agua a $50^{\circ} \mathrm{C}$ durante 60 minutos). El tratamiento T1 se recomienda respecto a los otros tratamientos porque mejoró la energía de germinación (48\%), tasa absoluta de germinación $(8,6$ semillas·día-1) e índice de germinación $(11,5)$.
\end{abstract}

Palabras clave: imbibición de semillas en agua, tasa absoluta de germinación, tratamientos de pre-germinación.

\begin{abstract}
Bitter gourd seeds present germination difficulties due to the thick and hard seed coat that surrounds the embryo. The objective of this study was to evaluate the effect of bitter gourd seed imbibition on germination energy, absolute rate of germination, and germination index. A completely randomized experimental design with three treatments was used: T0 (control, without imbibition), T1 (seed imbibition at $22^{\circ} \mathrm{C}$ during 24 hours), and T2 (seed imbibition at $50^{\circ} \mathrm{C}$ during 60 minutes). Treatment $\mathrm{T} 1$ is recommended over the other
\end{abstract}


treatments, because it improved germination energy (48\%), absolute rate of germination (8.6 seeds·day-1), and germination index (11.5).

Key words: Seed imbibition, absolute rate of germination, pre-germination treatment.

\section{INTRODUCCIÓN}

Momordica charantia L. es originaria de África, se utiliza en todo el mundo como planta alimenticia y medicinal, principalmente en India, China, Malaysia, Japón, y Sur América (Patil y Patil, 2011; Adesina et al., 2012). En la India hace parte de las más de 6000 plantas que tienen uso en la medicina natural y tradicional (Sharma et al., 2011). Es utilizada en medicina ayurvédica para el tratamiento de muchas enfermedades como diabetes, sarampión, fiebre y hepatitis, entre otras (Gupta et al., 2011). Recientemente, ha sido considerada como uno de los cinco cultivos que pueden salvar a la humanidad (Mc Creight et al., 2013).

M. charantia se propaga por semillas y por lo general, se siembra directamente, pero se pueden hacer trasplantes cuando las semillas son escasas. Principalmente, las semillas se caracterizan por la presencia de pequeños cotiledones encerrados por una testa dura y gruesa, lo que dificulta la germinación, por lo tanto, se han ensayado numerosos tratamientos pre-germinativos antes de la siembra para superar las limitaciones impuestas por la testa de la semilla (Jayasinha, 1999; Aye et al., 2004; Rahman et al., 2014; Saleem et al., 2014).

Entre los tratamientos físicos y químicos de pregerminación utilizados para mejorar la germinación y establecimiento de $M$. charantia se encuentran la exposición a campos eléctricos y magnéticos (Mahajan y Pandey, 2015), golpe y perforación de las semillas, escarificación de cubiertas, refrigeración, corte de embriones en mitades (Ebert et al., 2014) e inmersión en sustancias como $\mathrm{Si}, \mathrm{KNO}_{3}$, $\mathrm{KCl}, \mathrm{K}_{2} \mathrm{HPO}_{4}, \mathrm{KH}_{2} \mathrm{PO}_{4}, \mathrm{MgSO}_{4}, \mathrm{CaCl}_{2}, \mathrm{NaCl}, \mathrm{Na}_{2} \mathrm{SeO}_{3}$, $\mathrm{AG}_{3}, \mathrm{~B}(\mathrm{OH})_{3}, \mathrm{H}_{2} \mathrm{SO}_{4}$, Carbendazim, agua y agua caliente (Ashrafuzzaman et al., 2010; Wang et al., 2010; Islam et al., 2012; Rahman et al. 2014; Mehta et al., 2014).
Uno de los métodos más versátiles para reactivar los procesos bioquímicos en la fisiología de la germinación de las semillas de M. charantia, es la imbibición en agua, que es interesante en vista de las siguientes ventajas: germinación rápida y uniforme, aumento en la velocidad de emergencia de las plántulas, uso de una tasa de siembra más baja, no hay necesidad de riego después de la siembra y la posibilidad de descartar semillas muertas y de bajo vigor antes de la siembra (Thirusenduraselvi y Jerlin, 2007).

La práctica de imbibición en agua, ha dado también resultados satisfactorios en semillas de otras especies como Solanum melongena L. (Barraza, 2013), con tratamientos durante 72 horas para alcanzar 96\% de germinación, en comparación con semillas no tratadas (71\%). En el caso de $M$. charantia, Barraza (2015) obtuvo 76\% de germinación, con la imbibición de las semillas en agua durante 24 horas, en comparación con $62 \%$ para el tratamiento de control (sin imbibición), del cual se reporta como promedio el $65 \%$ de acuerdo con Thapa (2011).

Por lo anterior, el objetivo de la presente investigación fue evaluar la respuesta que tienen las semillas de $M$. charantia respecto a su energía de germinación, tasa absoluta de germinación e índice de germinación, en relación con el tratamiento de imbibición en agua (práctica común de los agricultores, Kanwar et al., 2014) e imbibición en agua a $50^{\circ} \mathrm{C}$.

Además, se identificaron las principales fases fenológicas del estado de germinación, a través de la utilización de la escala BBCH (Meier, 2001), con la cual se hace una codificación uniforme de identificación fenológica para todas las especies de plantas mono y dicotiledóneas, que constituye un vocabulario científico común que puede 
ser entendido de la misma manera por todas las personas vinculadas al estudio e investigación en diferentes campos del conocimiento.

\section{MATERIALES Y MÉTODOS}

La investigación se realizó en la Universidad de Córdoba, Facultad de Ciencias Agrícolas, Montería, Colombia, en el año 2015. Se hizo un bio-ensayo de germinación en el Laboratorio de Fisiología Vegetal, con semillas de balsamina de la variedad "criolla" que fueron recolectadas en cultivos del Campo Experimental de Hortalizas.

Se utilizó el diseño Completamente al Azar, con tres tratamientos y cuatro repeticiones. Los tratamientos fueron los siguientes: T0 (control, sin imbibición en agua), T1 (semillas embebidas en agua a $22^{\circ} \mathrm{C}$, durante 24 horas) y T2 (semillas embebidas en agua a $50^{\circ} \mathrm{C}$ durante 60 minutos). Cada repetición constó de 25 semillas colocadas en patrón circular sobre papel filtro humedecido con $15 \mathrm{~mL}$ de agua destilada estéril, en una placa Petri de vidrio, de 90mm de diámetro (ISTA, 2011).

Curva de imbibición de agua. En cada tratamiento, las semillas se pesaron cada seis horas, desde el inicio del bio-ensayo hasta las 96 horas posteriores, con el fin de determinar la cantidad de agua embebida (peso final menos peso inicial), expresada en porcentaje (Mehta et al., 2014).

Energía de germinación (EG). Para cada tratamiento se determinó la energía de germinación, mediante la siguiente ecuación (Hartmann et al., 2010):

$$
E G=\frac{2}{3} T G
$$

Donde:

$\mathrm{EG}=$ energía de germinación, expresada en porcentaje de semillas germinadas respecto al total.

$\mathrm{TG}=$ tiempo total de germinación = IG+PG, expresado en días, donde:
$\mathrm{IG}=$ tiempo de inicio de la germinación, $\mathrm{PG}=$ periodo de germinación (tiempo transcurrido entre el inicio y el final de la germinación).

Para determinar IG se cuantificó el tiempo de inicio de la germinación (días); para cuantificar PG se cuantificó el tiempo transcurrido entre el inicio y el final de la germinación (días).

Velocidad absoluta de germinación (VAG). Para calcular la VAG, se tomaron datos del porcentaje de germinación (Pg), mediante el conteo diario de semillas con emergencia de radícula hasta los seis días después de la siembra y se utilizó la siguiente ecuación (Islam et al., 2012):

$P g=\frac{\text { Número de semillas con emergencia de radícula }}{\text { Número de semillas sembradas }} \times 100$

Debido a que el Pg se ajustó al modelo logístico, $y=$ $A /\left(1+B^{*} e^{-c x}\right)$, la velocidad absoluta de germinación (VAG) se calculó como la primera derivada de dicho modelo, $y^{\prime}$ (Calvo et al., 1994; Barraza, 2015), mediante el procedimiento PROC NLIN en el software SAS versión 9.1.3 (SAS Institute, 2008):

$$
y^{\prime}=\left(A * B * C * e^{-C X}\right)^{2}
$$

Donde:

$y=$ porcentaje de germinación; $A$ : máximo valor observado en el porcentaje de germinación; $B$ : no tiene significado biológico y solo toma lugar en el tiempo inicial, cuando $x=0 ; x=$ tiempo (días) después de la siembra; $C=$ parámetro relacionado con el valor de $x$ para el punto de inflexión; $e=2,718281828$. Para determinar la aproximación de los modelos a los datos experimentales, se calculó el coeficiente de determinación $\left(\mathrm{R}^{2}=\right.$ proporción de la variabilidad presente en los datos que es explicada por el modelo de ANDEVA, mediante la siguiente ecuación:

$\mathrm{R}^{2}=\mathrm{SCt} / \mathrm{SCT}$, donde: 
$\mathrm{SCt}=$ suma de cuadrados entre tratamientos

SCT = suma de cuadrados total

Índice de germinación. Para calcular el índice de germinación ( $I G$, sin dimensiones), se aplicó la siguiente fórmula (Islam et al., 2012):

$$
I G=\frac{t i N i}{S}
$$

Donde:

ti : i-ésimo número de días después de la siembra

$\mathrm{Ni}$ = i-ésimo número de semillas germinadas

$S=$ número total de semillas utilizadas

Codificación del estado de germinación. Se tomó como base, el tratamiento T0 (control, sin imbibición en agua) y se asignaron códigos de 2 y 3 dígitos a las principales fases fenológicas del estado de germinación, de acuerdo con la escala $\mathrm{BBCH}$ (Meier, 2001).
Para el análisis estadístico de las variables energía de germinación, porcentaje de germinación, velocidad absoluta de germinación e índice de germinación, se hizo el ANDEVA y la prueba de comparación de medias de Tukey para establecer diferencias significativas entre medias de tratamientos a un determinado tiempo $(\mathrm{p} \leq 0,05)$, mediante el software SAS (PROC ANDEVA).

Para establecer la relación entre el porcentaje de germinación (Pg) y el índice de germinación (IG), se graficó Pg en función de IG y también se obtuvieron las respectivas líneas de tendencia mediante el programa de software Microsoft Excel ${ }^{\circledR} 2016$.

\section{RESULTADOS Y DISCUSIÓN}

Curva de imbibición de agua. La curva de imbibición de agua de las semillas de M. charantia para todos los tratamientos, presentó un patrón trifásico (Figura 1 y Tabla 1), lo cual coincidió con resultados de Mehta et al. (2014).

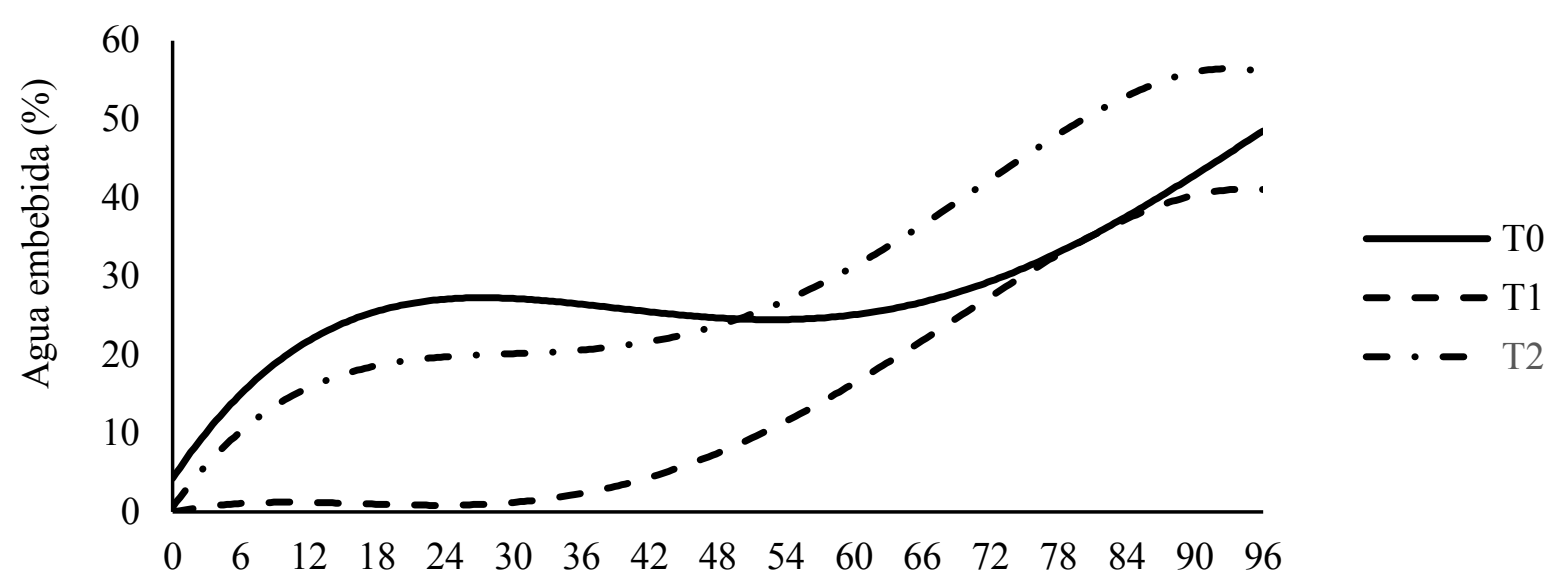

Tiempo después de la siembra (horas)

Figura 1. Curva de imbibición de semillas de balsamina variedad "criolla", en diferentes tratamientos de imbibición en agua. T0 (control, sin imbibición en agua), T1 (semillas embebidas en agua a $22{ }^{\circ} \mathrm{C}$, durante 24 horas) y T2 (semillas embebidas en agua a $50{ }^{\circ} \mathrm{C}$ durante 60 minutos). 
En términos generales, la Figura 1 muestra que el porcentaje de agua embebida por las semillas, se incrementó a medida que transcurrió el tiempo. De acuerdo con la Tabla 1, en la Fase I tuvo lugar una rápida toma de agua por parte de las semillas, lo que concordó con lo indicado por Pêgo et al. (2012). En el caso de T1, la cantidad de agua embebida fue menor que la de los tratamientos T0 y T2 (Tabla 1), lo que posiblemente se explica en términos de lo indicado por Ünal et al. (2013) en el sentido de que la imbibición en agua por 24 horas a que fueron sometidas las semillas, favoreció su hidratación inicial, que pudo ocasionar menor gasto energético y ayudar a la activación temprana de enzimas, requerida para iniciar la germinación.

Durante la Fase II, también llamada meseta o estacionaria de acuerdo con Pêgo et al. (2012), se observó un comportamiento constante en la imbibición de agua de las semillas, con poca ganancia de peso (Tabla 1). Esta fase fue variable de acuerdo con los tratamientos y según lo indicado por Mc Donald (2008) y Pêgo et al. (2012) puede variar también entre especies, cultivares o lotes de semillas, debido a diferencias genéticas o la calidad fisiológica.

Con respecto a la fase III, para T1 y T2, la imbibición de agua comenzó de manera más temprana que para T0 (Tabla 1 ).
Energía de germinación y porcentaje de germinación. Para T1, ambas variables mostraron valores significativamente más altos que en T0 y T2 (Tabla 2 y Tabla 3). Estos resultados indican la posibilidad de que la imbibición de semillas de $M$. charantia en agua a $22^{\circ} \mathrm{C}$ durante 24 horas, haya contribuido a la activación metabólica celular y aumento de la actividad enzimática, favoreciendo la germinación de acuerdo con lo reportado por Mehta et al., (2014) y Kanwar et al. (2014). Además de lo anterior, la imbibición de las semillas en agua, pudo haber contribuido en el crecimiento del embrión y ablandamiento de la testa, lo cual podría jugar un papel crucial acelerando la germinación, en concordancia con lo indicado por Lin y Sung (2000).

Tabla 2. Comparación de medias de energía de germinación (EG), porcentaje de germinación (Pg) y velocidad absoluta de germinación (VAG) de semillas de balsamina variedad "criolla", en diferentes tratamientos de imbibición en agua.

\begin{tabular}{cccc}
\hline Tratamiento & $\begin{array}{c}\text { EG } \\
\text { (\%) }\end{array}$ & $\begin{array}{c}\text { Pg } \\
\text { (\%) }\end{array}$ & $\begin{array}{c}\text { VAG } \\
\text { (semillas·día }\end{array}$ \\
\hline T0 & $40 \mathrm{~b}$ & $62 \mathrm{c}$ & $4,8 \mathrm{c}^{\dagger}$ \\
T1 & $48 \mathrm{a}$ & $76 \mathrm{a}$ & $9,6 \mathrm{a}$ \\
T2 & $36 \mathrm{c}$ & $64 \mathrm{~b}$ & $6,1 \mathrm{~b}$ \\
\hline
\end{tabular}

T0: (control, sin imbibición en agua), T1 (semillas embebidas en agua a $22^{\circ} \mathrm{C}$, durante 24 horas), T2 (semillas embebidas en agua a $50^{\circ} \mathrm{C}$ durante 60 minutos). ${ }^{\dagger}$ Valores de los tratamientos con la misma letra en las columnas, son iguales (Tukey; $\mathrm{p} \leq 0,05$ ).

Tabla 1. Cantidad de agua embebida (\%) y tiempo (h) de inicio y finalización de las fases de la germinación de semillas de balsamina variedad "criolla", en diferentes tratamientos de imbibición en agua

\begin{tabular}{c|cccc|cccc|cccc}
\hline \multirow{2}{*}{ Tratamiento } & \multicolumn{4}{|c|}{ Fase I } & \multicolumn{4}{c|}{ Fase II } & \multicolumn{4}{c}{ Fase III } \\
& \multicolumn{2}{|c}{ IA (\%) } & \multicolumn{2}{c|}{ Tiempo (h) } & \multicolumn{2}{|c}{ IA (\%) } & \multicolumn{2}{c}{ Tiempo (h) } & \multicolumn{3}{c}{ IA (\%) } & Tiempo (h) \\
& Inicio & Fin & Inicio & Fin & Inicio & Fin & Inicio & Fin & Inicio & Fin & Inicio & Fin \\
\hline T0 & 0 & 23,7 & 0 & 18 & 25,1 & 26,9 & 24 & 60 & 27,4 & 49,1 & 66 & 96 \\
T1 & 0 & 2,0 & 0 & 30 & 3,3 & 3,4 & 36 & 42 & 6,6 & 42,2 & 48 & 96 \\
T2 & 0 & 20,6 & 0 & 30 & 21,6 & 21,8 & 36 & 42 & 24,1 & 56,9 & 48 & 96 \\
\hline
\end{tabular}

IA: imbibición de agua; T0 (control, sin imbibición en agua), T1 (semillas embebidas en agua a $22{ }^{\circ} \mathrm{C}$, durante 24 horas), T2 (semillas embebidas en agua a $50^{\circ} \mathrm{C}$ durante 60 minutos). 
Tabla 3. Cuadrados de medios (cm) del ANDEVA para energía de germinación (EG), porcentaje de germinación (Pg) y velocidad absoluta de germinación (VAG) de semillas de balsamina variedad Criolla, en diferentes tratamientos de imbibición en agua.

\begin{tabular}{lllll}
\hline & \multicolumn{4}{c}{ cm } \\
\hline F.V & GL & EG & Pg & VAG \\
Tratamiento & 2 & 149,33 & 22,93 & 24,40 \\
Error & 9 & 71,11 & 12,40 & 0,12 \\
CV & & 1,2 & 1,4 & 1,7 \\
Significancia & & $*$ & $*$ & $*$ \\
\hline
\end{tabular}

FV: fuente de variación, GL: grados de libertad, CV: coeficiente de variación, *: diferencias estadísticas significativas (Tukey; $\mathrm{p} \leq 0,05$ ).

Con respecto a la energía de germinación, en general, se presentaron valores por debajo del $50 \%$, los cuales se pueden considerar bajos (Tabla 2), teniendo en cuenta que para el tratamiento control (sin imbibición en agua) Mahajan y Pandey (2015) reportan un valor de $60 \%$. Sin embargo, los mayores valores obtenidos para T1, en comparación con los de T0 y T2, muestran que la imbibición de semillas en agua durante 24 horas está relacionado con la toma de agua por las semillas y su influencia en la capacidad de germinación, como ha sido reportado para M. charantia por Mahajan y Pandey (2015).
Respecto al porcentaje de germinación, para el tratamiento T0 los resultados obtenidos coinciden con los de Islam et al. (2012), y para el caso de los tratamientos T1 y T2 los valores obtenidos se encuentran por debajo de los indicados por estos mismos autores, quienes reportan 66\% para el tratamiento con imbibición en agua por 24 horas y $96 \%$ para imbibición en agua a $50^{\circ} \mathrm{C}$ durante 60 minutos.

Velocidad absoluta de germinación. Se presentaron diferencias estadísticas significativas entre tratamientos (Tabla 2 y Tabla 3). En la Figura 2 se observa que hubo un patrón de distribución normal, en forma de campana, que concuerda con los resultados de Mehta et al. (2014), con mayores valores a los seis días después de la siembra (Figura 2). Los modelos matemáticos obtenidos para la explicación de esta variable se presentan en la Tabla 4.

Índice de germinación. Esta variable incrementó a medida que pasó el tiempo, lo que coincidió con lo indicado por Islam et al. (2012), Ünal et al. (2013) y Mehta et al. (2014). En general, hubo valores más altos para T1, cuyos valores promedios superaron en grado significativo a los obtenidos con los tratamientos T0 y T2 desde los 4 hasta los 6 días después de la siembra (Tabla 5).

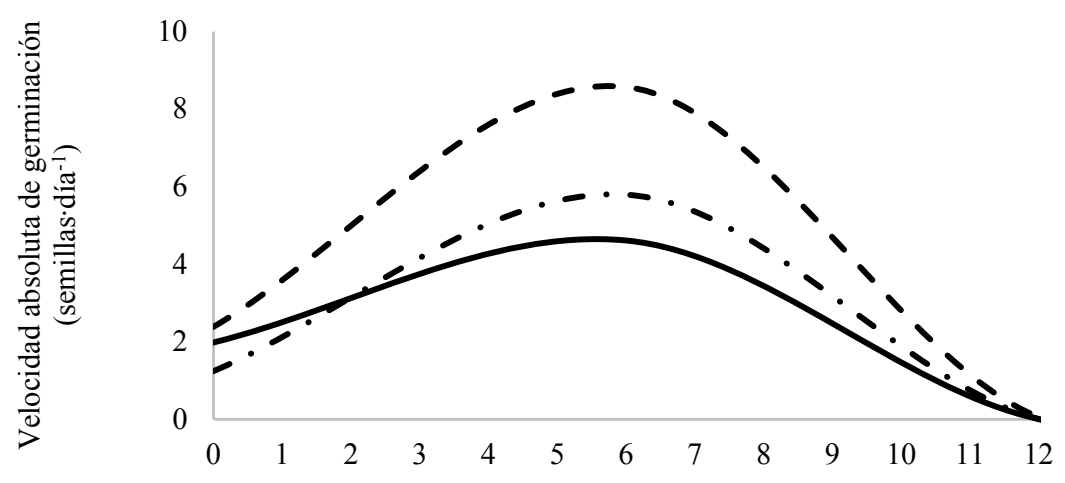

Tiempo después de la siembra (días)

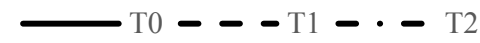

Figura 2. Velocidad absoluta de germinación de semillas de balsamina variedad "criolla", con diferentes tratamientos de imbibición en agua. T0 (control, sin imbibición en agua), T1 (semillas embebidas en agua a $22^{\circ} \mathrm{C}$, durante 24 horas) y T2 (semillas embebidas en agua a $50^{\circ} \mathrm{C}$ durante 60 minutos). 
Tabla 4. Modelos matemáticos para estimar la velocidad absoluta de germinación de semillas de balsamina variedad "criolla" en diferentes tratamientos de imbibición en agua.

\begin{tabular}{cc}
\hline Tratamiento & Modelo matemático \\
\hline T0 & $y^{\prime}=\left(68,83 * 33,03 * 1,01 * \mathrm{e}^{-1,01 x}\right) /\left(1+33,03 * \mathrm{e}^{-1,01 x}\right)^{2}$ \\
T1 & $y^{\prime}=\left(86,44 * 30,16 * 0,89 * \mathrm{e}^{-0,89 x}\right) /\left(1+30,16 * \mathrm{e}^{-0,89 \mathrm{x}}\right)^{2}$ \\
$\mathrm{~T} 2$ & $y^{\prime}=\left(71,93 * 61,38 * 1,09 * \mathrm{e}^{-1,09 x}\right) /\left(1+61,38 * \mathrm{e}^{-1,09 x}\right)^{2}$ \\
\hline
\end{tabular}

T0: (control, sin imbibición en agua), $\mathrm{T} 1$ (semillas embebidas en agua a $22^{\circ} \mathrm{C}$, durante 24 horas), $\mathrm{T} 2$ (semillas embebidas en agua a $50^{\circ} \mathrm{C}$ durante 60 minutos).

Tabla 5. Comparación de medias del Índice de germinación (IG) de semillas de balsamina variedad "criolla" en diferentes tratamientos de imbibición en agua.

\begin{tabular}{cccc}
\hline Tiempo (d) & T0 & T1 & T2 \\
\hline 0 & $0,0 \mathrm{a}$ & $0,0 \mathrm{a}$ & $0,0 \mathrm{a}$ \\
3 & $2,3 \mathrm{a}$ & $2,3 \mathrm{a}$ & $2,0 \mathrm{a}$ \\
4 & $4,8 \mathrm{~b}$ & $5,3 \mathrm{a}$ & $4,3 \mathrm{~b}$ \\
5 & $7,9 \mathrm{~b}$ & $8,4 \mathrm{a}$ & $7,4 \mathrm{~b}$ \\
6 & $10,5 \mathrm{~b}^{\dagger}$ & $11,5 \mathrm{a}$ & $10,0 \mathrm{~b}$ \\
\hline
\end{tabular}

T0 (control, sin imbibición en agua), T1 (semillas embebidas en agua a $22^{\circ} \mathrm{C}$, durante 24 horas), $\mathrm{T} 2$ (semillas embebidas en agua a $50{ }^{\circ} \mathrm{C}$ durante 60 minutos). ${ }^{\dagger}$ Valores de los tratamientos con la misma letra son iguales (Tukey; $\mathrm{p} \leq 0,05$ ).
El índice de germinación tuvo una relación lineal significativa con el porcentaje degerminación(Figura $3)$, lo que coincidió con lo reportado por Islam et al. (2012). La variación obtenida en el porcentaje de germinación para todos los tratamientos, puede ser explicada en términos del índice de germinación, que de acuerdo con Thirusenduraselvi y Jerlin (2007) y Chimonyo y Modi (2013) es una característica intrínseca y de calidad fisiológica de las semillas, que está reforzada por los nutrientes, reguladores de crecimiento y sustancias bioactivas liberadas cuando las semillas se embebieron en agua, y que participan en el ablandamiento de la testa.

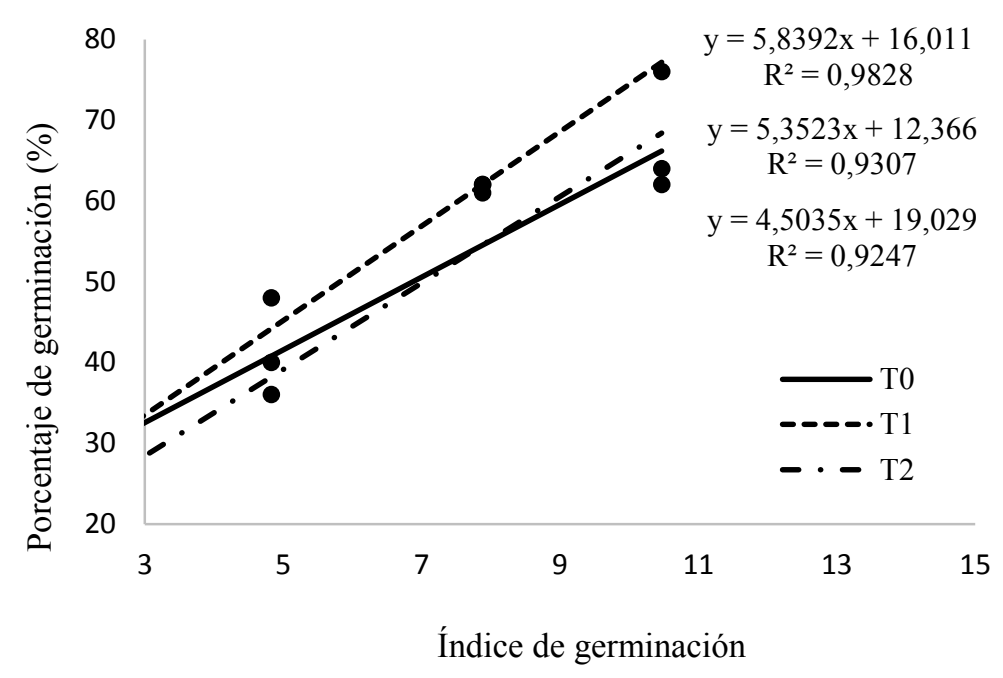

Figura 3. Relación entre el porcentaje de germinación y el índice de germinación de semillas de balsamina variedad "criolla", en diferentes tratamientos de imbibición en agua. T0 (control, sin imbibición en agua), T1 (semillas embebidas en agua a $22^{\circ} \mathrm{C}$, durante 24 horas), $\mathrm{T} 2$ (semillas embebidas en agua a $50^{\circ} \mathrm{C}$ durante 60 minutos). 
Codificación del estado de germinación. En la Tabla 6 y Figura 4 se muestran los códigos de la escala $\mathrm{BBCH}$ asignados al estado fenológico principal 0 (germinación). Se pudo observar que después de sembrar las semillas comenzó la imbibición, que en concordancia con Kanwar et al. (2014) es el primer proceso que debe ocurrir antes de la germinación. Finalizada la imbibición hubo una serie de cambios morfológicos graduales y progresivos en tamaño que explican de acuerdo con Ebert et al. (2014) que el embrión de la semilla reanudó su crecimiento para transformarse en una plántula completa con los siguientes órganos: radícula, hipocotilo y hojas cotiledonales.

En la Figura 4 se puede observar que las hojas cotiledonales se elevaron por encima de la superficie de siembra, lo que correspondió al tipo de germinación epigea. Este evento indicó la emergencia, con la cual culminó el estado fenológico principal de germinación (Tabla 6).

\section{CONCLUSIONES}

El tratamiento de imbibición de semillas de $M$. charantia en agua a $22^{\circ} \mathrm{C}$ durante 24 horas produjo mejor calidad fisiológica de las semillas con un mayor porcentaje de germinación, energía de germinación y velocidad absoluta de germinación.

El índice de germinación tuvo relación lineal con el porcentaje de germinación, con independencia del tratamiento.

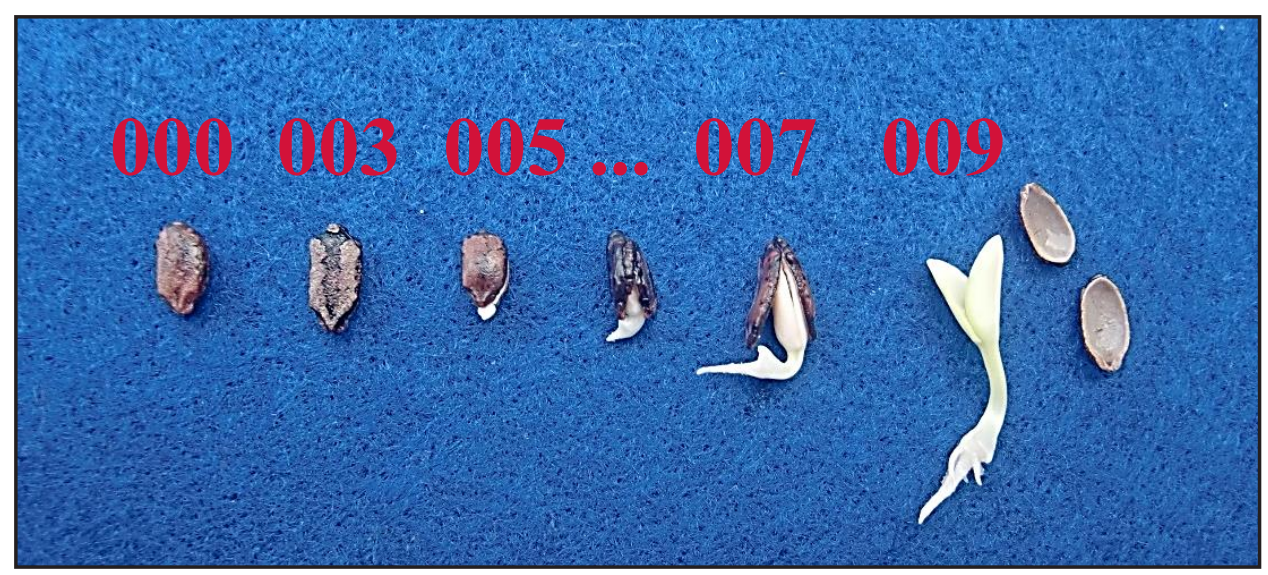

Figura 4. Códigos de la escala BBCH asignados al estado fenológico principal 0 (germinación) de semillas de balsamina variedad Criolla.

Tabla 6. Códigos de la escala BBCH para el estado fenológico principal 0 (germinación) de semillas de balsamina variedad "criolla".

\begin{tabular}{cclc}
\hline $\begin{array}{c}\text { Código de } \\
\text { 2 dígitos }\end{array}$ & $\begin{array}{c}\text { Código de } \\
\text { 3 dígitos }\end{array}$ & \multicolumn{1}{c}{ Descripción } & $\begin{array}{c}\text { Tiempo } \\
\text { (h) }\end{array}$ \\
\hline 00 & 000 & Semilla seca & 0 \\
01 & 001 & Comienzo de la imbibición de la semilla & 1 \\
03 & 003 & Imbibición completa de la semilla & 36 \\
05 & 005 & Radícula emergida de la semilla & 66 \\
07 & 007 & Hipocotilo con cotiledones rompiendo a través de la cubierta de la semilla & 81 \\
09 & 009 & Emergencia: cotiledones rompiendo a través de la superficie del suelo & 93 \\
\hline
\end{tabular}




\section{REFERENCIAS BIBLIOGRAFÍCAS}

ADESINA, J.M.; AFOLABI, L.A.; OFUYA, T.I. 2012. Evaluation of insecticidal properties of Momordica charantia in reducing oviposition and seed damaged by Callosobruchusma.culatus (Fab.) Walp. J Agric Sci Technol. 8:493 - 499 .

ASHRAFUZZAMAN, M.; ISMAIL, M.; FAZAL, K.; UDDIN, M.; PRODHAN, A. 2010. Effect of GABA application on the growth and yield of bitter gourd (Momordica charantia L.). Int J Agr Biol. 12:129 - 132.

AYE, M.M.; SUNG, Y.; CHANG, W. 2004. Techniques inproved 'Ching Pi' bitter gourd (Momordica charantia L.) seed germination. Hort Nat Chung Hsing Un. 29:27 - 42 .

BARRAZA, F.V. 2013. Crecimiento y calidad morfológica de berenjena (Solanum melongena L.) en fase de semillero. Tem Agr. 8:7 - 20.

BARRAZA, F.V. 2015. Physical characteristics and germination of bitter gourd (Momordica charantia Linn.) seeds. Indian J Res. 4:3 - 5.

CALVO, R.; GONZÁLEZ, J.; PÉREZ, S. 1994. Manual de modelos no lineales en los ámbitos agronómico, ganadero y forestal. Ministerio de Agricultura, Pesca y Alimentación. I.N.I.A. Madrid. 110 p.

CHIMONYO, V.; MODI, A. 2013. Seed performance of selected bottle gourd (Lagenaria siceraria (Molina) Standl.). Amer J Exp Agr. 3:740 - 766.

EBERT, A.; HUANG, Y.; CHOU, Y. 2014. Bitter gourd germplasm diversity and seed research. pp. 1-34. En: Bitter Gourd Conference. Hyderabad, India.

GUPTA, M.; SHARMA, S.; GAUTAM, A.; BHADAURIA, R. 2011. Momordica charantia Linn. (karela): nature's silent healer. Int J Pharm Sci Rev Res. 11:32 - 37.

HARTMANN, H.; KESTER, D.; DAVIES, F.; GENEVE, R. 2010. Plant propagation. Principles and practices. $8^{\text {th }}$ edition. Prentice Hall, New Jersey. 915 p.

ISLAM, S.; MIA, A.; HOSSAIN, T.; AHMED, J.; KHAN, H. 2012. Priming on embryo emergence and seedling vigor of small fruited bitter gourd (Momordica cha- rantia L.) under suboptimal temperature. Int J Agr Sci Res. 2:1 - 10 .

ISTA. INTERNATIONAL SEED TESTING ASSOCIATION. 2011. Rules proposals for the international rules for seed testing 2011 Edition ISTA. Bassersdorf, Switzerland. $53 \mathrm{p}$.

JAYASINHA, P. 1999. Karawila. Momordica charantia. A literature survey. Medicinal and Aromatic Plant Series No. 3. Information Services Centre, Industrial Technology Institute, Colombo, Sri Lanka. 36 p.

KANWAR, R.; MEHTA, D.; LAL, M. 2014. Effect of seed priming on physiological parameters of aged and nonaged seeds of bitter gourd, Momordica charantia L. Int J Farm Sci. 4:24 - 32.

LIN, J.; SUNG, J. 2000. Presowing treatments that improving germination of bitter gourd seeds under suboptimal temperature. Seed Science and Tecnology. 29(1):39 - 50.

MAHAJAN, T.; PANDEY, 0. 2015. Effect of electric and magnetic treatments on germination of bitter gourd (Momordica charantia) seed. Int J Agr Biol. 17:351 356.

Mc CREIGHT. J.; STAUB, J.; WEHNER, T.; DHILLON, N. 2013. Gone global: familiar and exotic cucurbits have asian origins. Hort Sci. 48:1078 - 1089.

Mc DONALD, M. 2008. Physiology of seed germination. Consortium for International Seed Technology Training. Ohio State University. Columbus. 24 p.

MEHTA, D.; KANWAR, H.; THAKUR, A.; THAKUR, S.; THAKUR, K. 2014. Standardization of seed hydro-priming duration in bitter gourd, Momordica charantia L. Int J Bio-res St Man. 5:98 - 101.

MEIER, U. 2001. Growth stages of mono-and dicotyledonous plants. BBCH Monograph. Second edition. Federal Biological Research Centre for Agriculture and Forestry. Berlin and Braunschweig, Germany. 149 p.

PATIL, S.A.; PATIL, S.B. 2011. Toxicological studies of Momordica charantia Linn seed extracts in male mice. Int J Morph. 29:1212 - 1218. 
PÊGO, R.; GROSSI, J.; BARBOSA, J. 2012. Soaking curve and effect of temperature on the germination of daisy seeds. Hort Bras. 30:312 - 316.

RAHMAN, M.; ISLAM, M.; SHAHEB, M.; SARKER, P.; NESSA, A.; SARKER, M. 2014. Influence of sowing date on quality seed production of bitter gourd. Int J Sust Crop Prod. 9:17 - 21.

SAS INSTITUTE INC. 2008. Statistical analysis system. The $\mathrm{SAS}^{\odot}$ system for Windows ${ }^{\odot}$ version 9.1.3. The Power to Know. Cary, NC, EEUU.

SALEEM, M.; SAJID, M.; AHMED, Z.; AHMED, S.; AHMED, N.; ISLAM, M. 2014. Effect of seed soaking on seed germination and growth of bitter gourd cultivars. Journal of Agr Vet Sci. 6:7 - 11.

SHARMA, S.; TANDON, SH.; SEMWAL, B.; SINGH, K. 2011. Momordica charantia Linn: A comprehensive review on bitter remedy. J Pharm Res 0p. 1:42 - 47.

THAPA, M. 2011. Quality analysis of marketed seeds of some crops in Nepal. Agr J Nepal. 2:149 - 156.

THIRUSENDURASELVI, D.; JERLIN, R. 2007. Effect of pre-germination treatments on the emergence percentage of bitter gourd cv. CO 1 seeds. Trop Agr Res Ext. 10:88 - 90 .

ÜNAL, H.; ALPSOY, H.C.; AYHAN, A. 2013. Effect of the moisture content on the physical properties of bitter gourd seed. Int Agroph. 27:455 - 461.

WANG, X.; OU-YANG, CH.; FAN, Z.; GAO, SH.; CHEN, F.; TANG, L. 2010. Effects of exogenous silicon on seed germination and antioxidant enzyme activities of Momordica charantia under salt stress. J An Pl Sci. 6:700 - 708. 